\title{
Community paramedicine: Higher education as an enabling factor
}

\author{
${ }^{1}$ Peter O'Meara PhD, ${ }^{2}$ Michel Ruest, ${ }^{3}$ Christine Stirling PhD \\ Affiliations: \\ ${ }^{1}$ LaTrobe University, Victoria, Australia. \\ 2 Renfrew County Paramedic Service, \\ 3 University of Tasmania, Tasmania, Australia
}

\section{SUMMARY}

The aim of this case study was to describe one rural community paramedic model and identify enablers related to the implementation of the model. It was undertaken in the County of Renfrew, Ontario, Canada where a community paramedicine role has emerged in response to demographic changes and broader health system reform. Qualitative data was collected through direct observation of practice, informal discussions, interviews and focus groups.

The crucial role of education in the effective and sustainable implementation of the community paramedicine model was identified as one of four enablers. Traditional paramedicine education programs are narrowly focused on emergency response, with limited education in health promotion, aged care and chronic disease management. Educational programs hoping to include a wider range of topics face the twin challenges of an already crowded curriculum and predominately young students who fail to see the relevance of community primary care content.

A closer match between the paramedicine curriculum and the emerging roles of paramedics, whether they are community paramedics, extended care paramedics, or as yet unformed roles is needed if paramedics are to become valued members of the health care team.

\section{Keywords}

Paramedic, emergency medical technicians, rural health, education

\section{INTRODUCTION}

Paramedics are increasingly becoming first line primary health care providers in small rural communities and as a result they are developing additional professional responsibilities throughout the cycle of care (1-3). Throughout the world, Emergency Medical Services (EMS) are increasingly required to provide a wider range of clinically-focused services than in the past and to also be more accountable than ever before. In parallel with these pressures, paramedics are developing new professional roles and identities that are broader and more sophisticated than that of the traditional emergency response model (46). Rural paramedics, in particular, are increasingly working as the 'expert' in the discipline of acute primary health care in unstructured community settings (2).

The emergence of more complex professional paramedic roles raises questions related to community and professional expectations and the identification of enabling factors associated with the sustainability of emerging models, such as community paramedicine roles. These factors may include the degree to which paramedicine is integrated within the health system, effectiveness of clinical governance systems, educational foundations for paramedics, and the development of appropriate clinical pathways for patients and clients (7). This paper addresses paramedicine education as one of these enabling factors. 
New and expanded paramedicine roles are emerging in response to an increasingly ageing population with more complex morbidities, higher community expectations and the contraction of some other health services (8). For instance, many smaller, rural hospitals have limited or no emergency department or maternity services, while many family physicians are unwilling or unable to provide house calls to isolated patients for a variety of reasons, including the threat of violence $(9,10)$. As an example, reports indicate that in Australia less than half of the country's family physicians regularly conduct home visits (11). Emergency Medical Services and paramedics throughout the world are consequently stepping into these gaps and facing the twin challenges of changing levels of clinical practice and greater demands for accountability.

As a result, EMS and paramedics are increasingly facing higher levels of organizational and professional scrutiny through mechanisms such as clinical governance systems and occupational registration requirements (12-14). In their patient focused interactions with other health professionals they need to balance specialized expertise with their inherent role and education limitations. This working environment of paramedics is unique in the health system.

In 2005, the 1st International Roundtable on Community Paramedicine was held in Nova Scotia, Canada. This loosely-bound group has since developed a definition of community paramedicine that is gaining currency around the world. To reduce the potential for confusion their definition was adopted for this study.

Community Paramedicine is a model of care whereby paramedics apply their training and skills in "non-traditional" community-based environments, often outside the usual emergency response and transportation model. The community paramedic practices within an "expanded scope", which includes the application of specialized skills and protocols beyond the base paramedic training. The community paramedic engages in an "expanded role" working in non-traditional roles using existing skills.(15)

The aim of this case study was to describe a Canadian community paramedic model and to identify enablers related to successful implementation. This paper focuses on education as an enabler, with the others to be reported in detail elsewhere.

\section{METHODS}

\section{Setting}

This case study was undertaken in the County of Renfrew, Ontario, Canada where a community paramedic role has emerged in response to demographic changes and broader health system reform. The County was formed in 1861 and is made up of a number of small to medium sized towns, including Arnprior, Eganville, Pembroke, Renfrew, Barry Bay, Petawawa and Deep River, along with many other small villages and settlements. It sits along the boundary between the provinces of Ontario and Quebec and is largely defined by the Ottawa River.

The County of Renfrew Paramedic Service was formed in January 2005 as part of a Provincewide re-organization of paramedic services that locates service delivery at a municipal level and regulation at a provincial level. The Paramedic Service is part of the County's Emergency Services Department. It is financially and structurally separate to the provincial health system. The service's current model is an advanced life support system provided by Advanced Care Paramedics and Primary Care Paramedics, as defined under the Canadian Occupational Competency Profile (16), staffing seven vehicles 24 hours a day, seven days a week deployed from seven leased Ambulance Base Stations (17). At the time of the study, there were another three 12 hour-day shift crews deployed across the County.

\section{Research Approach}

Building on previous research in Australia (2), a range of qualitative methods were used to validate the professional boundaries of community paramedics and identify enabling factors associated with successful community paramedicine programs. The La Trobe University Human Research Ethics Committee approved the research (FHEC12/8).

Descriptions of the emerging paramedic roles were sought from managers, with the other data collected through a combination of direct observation of practice, informal discussions, interviews and focus groups. This qualitative approach from Stake (18) allowed the capture of data encompassing the richness and diversity of the community paramedicine role within a natural setting that allowed issues to be studied in depth. It also placed practice within the wider community context (19). Participants in the case study were recruited through purposive sampling and included:

- Community members, including patients, family and carers 
- Paramedics and EMS managers from the County of Renfrew and Greater Ottawa area

- Paramedic educators in the Province of Ontario

- Physicians, nurse practitioners and other health care providers who interact with community paramedics

- Expert informants, such as health economists and health service managers

Three focus groups of between 10 and 20 participants and 11 interviews with 14 participants were conducted using purposive sampling to draw in a range of respondents including EMS managers, paramedics, other health professionals and community members. Three facilitators, with backgrounds in paramedicine, nursing and health services research carried out the interviews and two of the investigators facilitated the focus groups. Focus groups and interviews were used because of their ability to encourage detailed, emotive responses, unconstrained by specific questions of a survey, to the points raised by the facilitators. It was an opportunity to collate common issues or expectations and provide a stimulating environment to encourage specific contributions.

The research team used the paramedicine domains of practice identified in an Australian study model to locate the elements of the specific community paramedicine program and to develop the focus group and interview questions. This approach reduced the risk of facilitator bias. These questions focused on the role of community paramedics in terms of community engagement, emergency response, situated practice and primary health care (2). There were no targeted questions that related to the education and training of community paramedics.

The focus group discussions and the expert informant interviews were recorded and transcribed, with each transcript being coded and analysed using classic thematic analysis techniques through manual methods consistent with the recommendations of Strauss and Corbin (20). Using inductive thematic analysis enabled identification of common themes within the qualitative data and the building of an explanatory theory, without the constraint of having to establish how these themes link together or explain all facets of the data (21).

Complementing the focus groups and interviews was field observation of the community paramedic in a natural setting - often described as naturalistic research (18). This type of qualitative research is distinctly different from observational studies used in epidemiology as it involves the systematic, detailed observation of behaviour and talk (22). Informal discussions with participants also formed an important component of this observational phase of the data collection process and helped establish the general perception pattern of the community paramedicine program (19). The advantage of using this approach was that it shone a light on any discrepancies between rhetoric and reality. For this reason observational methods were well suited to this case study of a community paramedicine program. This source of data facilitated a richer understanding of the behaviours and interactions of community paramedics in their natural setting. The resulting observations validated those data that had been gathered from documents, the EMS organization, interviews and focus groups.

During the field observation component of the research a record of community paramedics' practice was noted, along with the principal researcher's own feelings and responses. These notes were recorded during or immediately after events occurred (22). Analysis of the field notes was commenced during the data collection phase through content analysis, an iterative process of developing categories from the notes, testing them against concepts and other data, and refining them.

\section{RESULTS}

The Renfrew Community Paramedic Program developed in response to identified needs in a number of the communities within the County, primarily because of movements in populations and often a lack of services or physicians. The model emerged organically from existing structures and local needs and is built on strong partnerships between the Paramedic Service and other health and social services. A wide range of disparate community health initiatives have evolved into a coherent community paramedicine program. The program consists of four key elements:

- Ageing at Home Program

- Paramedic Wellness Clinics

- Ad hoc Home Visiting Program

- Paramedic Response Unit Program

The community paramedicine program has evolved over a number of years as the critical needs of the clients and specific communities have emerged. Some of these have included the institution of strategies that assist clients at risk of falls, address the long-term problems associated with diabetes, and the challenge of supporting families dealing with the challenges associated with dementia and Alzheimer's. These strategies are consistent with developments in other parts of the Canada, United Kingdom and the United States of America $(6,23)$. This program exists 
because paramedics both recognised a need and had the flexibility to create new services.

So really the way that the community paramedic programs, and there are multiple programs within the umbrella here in the County, were developed was really from the paramedics themselves and from the service recognizing that there were all kinds of gaps in the system and that we needed to find a way to start to plug those gaps either formally or informally. (Participant 4)

... at least in part, one of the strengths of the Community Paramedic Program is that because it's sometimes rightly or wrongly not seen as part of the traditional health system people see them as nontraditional partners and you can actually consider doing things that are a bit more innovative. So it is sort of the flip side to being outside the system. (Participant 3)

Community paramedics identified they were working in new ways that used very different skill sets from the EMS norm. They were often looking at environmental, chronic disease and prevention issues.

... most of the times we are working proactively to nip the problems before they happen. So if we see an altered behaviour in a client you know because we know them like the back of our hand. I say to [the Community Paramedic] "Are they drinking, do they need a urine to go to the hospital?" (Participant 15)

You know maybe it is an anxiety issue with somebody they are just feeling a little under the weather and want to talk so it's half an hour of sitting in the living room chatting and seeing what is going on and trying to get an idea of the bigger picture and little things like that. So very much I would say my use of medical technology is almost secondary in my day to day functions throughout the program. It's always available to me, it is always in my vehicle but it can sometimes play very much a minor role in me being there and me being a part of the program. (Participant 9)

Communication skills were identified as an area of major importance in the community paramedic role and the most important area where paramedicine education could be most improved.

Communication is the big one. I think the fact that one of the roles of paramedics is that they do often go into homes so they are often used to that kind of setting and they are used to the dynamic of not working just with the patient but working with the family as well. ... It is not just paramedic to patient ... there are family members around as well. So they have to deal with all that plus they are getting a sense of the setting so I think that they come in with that knowledge.

So I think communication is the big thing because they not only have to get the information from the patient, observe the setting and then that communication has to be passed onto the primary practitioner or whether it is the CHC [Community Health Centre] or somebody else. ... their model of care is very much based on a holistic model so there is a lot of population health factors and those kinds of thing. So I think a knowledge of that is really imperative so if you are doing home visits and you are working with the $\mathrm{CHC}$. (Participant 7)

Building trust and long term relationships were seen as enabling factors underpinning the emerging role of community paramedicine. It may be that building these trusting relationships only requires a change in attitude amongst paramedics to build on the high level of trust that people already have in paramedics.

You have to build the trust and what I find really neat since I started this program with these guys is every one of these guys, whether it be a Medic or a PSW (Personal Support Worker) or a house keeper, they all get a really neat relationship. One of them with a certain client and then that's the trust and that is the start of the trust that we build. (Participant 15)

One interesting aspect of these trust building exercises with patients, families and other health professionals is that paramedics normally deal with patients on a very short-term basis and it is a very big departure for paramedics to form longterm relationships with patients and families.

That's right, and to seek out that longterm relationship ... [Community Paramedic] you have grown with the program obviously, like when you think about yourself coming straight out of training you are trained for an emergency response situation thank goodness, but to come into this program is a little different? Absolutely you are here for emergency 
response as well but you are here for as you said long term. (Participant 15)

Many of the participants identified that the establishment of the Renfrew County community paramedicine program was only the first step toward implementation and sustainability. Study participants were strongly of the view that the program needs to become institutionalized and not be dependent on individual champions to ensure its ongoing existence. There is clearly a need for structures and mechanisms to make the program sustainable and to maintain standards of safety.

From an educational perspective, the findings indicated that community paramedicine programs are more likely to succeed if paramedic education and training provides graduates with suitable breadth and depth of capabilities that move beyond the traditional paramedic emergency response competencies $(23,24)$. These conclusions are consistent with the experience of other community paramedic programs around the world where paramedics have consistently " ... received additional training above and beyond the scope of practice for a locally identified paramedic." (25) In Canada, a National Occupational Competency Profile for the Community Paramedic role is reportedly being developed by the Paramedic Association of Canada that will help inform educational programs for community paramedics.

The education and training of paramedics in the Province of Ontario is largely based in the Community College system with students undertaking Primary Care Paramedic (PCP) twoyear Diploma program that includes defined hours of theory, clinical activities in hospitals, and ambulance service practicum. Advanced Care Paramedics (ACP) undertake an additional one year of education and training in the Community College system. There are also Critical Care Paramedic programs offered to those staff involved in specialist roles such as air retrieval (26).

The PCP programs cover the following areas of study: (26)

- Anatomy and Physiology;

- Psychopathology/Crisis Intervention;

- Pharmacology;

- Health Care Communication;

- Medico-Legal Aspects;

- Physical Education;

- Patient Care Laboratory;

- Patient Care Theory;

- Emergency Medicine;

- Emergency Vehicle Operation;

- Medical Directives;

- Clinical Practicum; and
- Field Practicum.

Advanced Care Paramedics (ACP) in the Province of Ontario undertake a one-year Graduate Certificate programs that include: (26)

- Advanced Pharmacology;

- Advanced Care Skills (Cardiac, Airway Management, Respiratory, Medical Emergencies, Trauma);

- Professional Practice;

- Skills Practicum;

- Hospital Practicum; and

- Advanced Care Paramedic Ambulance Practicum.

Participants in this case study highlighted the highly technical nature of the existing educational paramedicine programs in the Province of Ontario that focus on emergency responses and acute medical conditions. Many expressed the view that to undertake and embrace the community paramedicine role, paramedics need a broader educational base, with more knowledge and skills in patient assessment, clinical decision-making, and a greater appreciation of the public health aspects of patient and community health and wellbeing.

The other thing is that they are trained for emergencies ... clinics that you are having or the Ageing at Home Program is not an emergency type situation, so anecdotally what I have heard is it's been a big learning curve for some of those paramedics going into homes establishing relationships with people because they certainly don't do that when they are just picking them up on the side of the road and bringing them in? (Participant 6)

While paramedicine education programs in the Province of Ontario include assessment and clinical decision-making related to emergency care, one community college educator made the point that an already crowded curriculum limited the capacity to provide a broader educational platform. A particular need for education related to health promotion and prevention was identified. Participants clearly described those areas of paramedicine education and training that could be improved, as well as acknowledging those areas of expertise that paramedics already have that may be useful in a community paramedicine role.

... there isn't any education on health determinants, social determinants, the actual structure of how the system works ... what kind of skills do we need to be able to possess or what skills do we need or what knowledge do we need to be able to successfully integrate, collaborate with our partners. (Participant 3) 
In relation to the community paramedicine role in the County of Renfrew and elsewhere the education and training challenge is twofold. Firstly, the curricula of the current paramedicine programs are already at full capacity with acute care topics and skills. And secondly, the widespread difficulty of convincing predominately young students that the role of future paramedics is more about community primary care practice than answering emergency calls $(24,27,28)$.

I mean a lot of people who come into my program are looking for the lights and sirens and trauma and excitement and when we start talking about something with a slower pace in community paramedicine, we take them back. ... a lot of students sort of walk in the door going, I want to drive fast, lights and sirens and car crashes and all that good stuff, and when you say, well in actual fact that's about five percent of your career, 95 percent of your career looks much more like community paramedicine. (Participant 10)

Some participants in this study indicated that they would like to see paramedicine programs evolve into four-year degree courses comparable to other health professional disciplines and paramedic programs in Canada and other parts of the world (29-33). One suggestion made was that community paramedicine could become a specialist stream within a degree program, incorporating public health subjects and some specific procedural skills. Those paramedics taking part in the Renfrew County Community Paramedicine Program already undertake a five day Geriatric Emergency Medical Service course.

This latter idea has obvious links to developments in the United States of America, where a community paramedicine curriculum has been developed that incorporates public health topics and specific procedural skills $(23,34)$. In Australia and New Zealand degree-level paramedicine programs incorporate many of these public health topics. These well-established higher education programs include a mix of three-year paramedicine degrees and some four-year double degrees in combination with Nursing or Public Health. It is expected that degree-level paramedicine qualifications will be mandatory in Australia and New Zealand by 2015 (3).

\section{DISCUSSION}

The findings from this case study provide a rich picture of the working environment of community paramedics and how their roles may develop as part of an integrated health system. A range of enablers were identified, including the crucial role of education in the effective and sustainable implementation of the community paramedicine model. The limitations of this case study though prevent any definitive conclusion or generalizations. The results are not generalizable to other services because participants were recruited from within one geographic area. This did allow the analysis to integrate knowledge of the professional ethos and program with participants' responses. Further studies are needed to validate the findings reported here.

Traditional paramedicine education programs are narrowly focused on emergency response, with limited education in cultural safety, health promotion, aged care and chronic disease management (24). In many paramedicine programs there is a lack of education in the social determinants of health and how the wider health system operates (29). The lack of cultural diversity amongst paramedics in many settings highlights the importance of a broadly-based education system that addresses the public and community health needs of communities (35).

Paramedicine programs that aspire to a broader curriculum that includes a wider range of topics and competencies face the challenges of an already crowded curricula and also overcoming the perceptions and expectations of predominately young students that paramedicine work is restricted to responding to emergencies. Convincing students and other stakeholders that community primary care content is relevant to the future practice of paramedicine within an integrated health system is a key policy challenge for educators, leaders of paramedic services and the paramedicine profession.

Amongst the short-comings of the current paramedicine education system in the Province of Ontario is that there is not a significant amount of education on health determinants, social determinants, or the structure of how the health system works. Students are not taught about where paramedicine services fit into the health system, or what kind of skills and knowledge paramedics need to possess to be able to successfully integrate and collaborate with other institutions or health professions. This is particularly important in community paramedicine programs where there is a strong emphasis on maintaining patients in their own homes.

A closer match between the paramedicine curriculum and the emerging roles of paramedics, whether they are community paramedics, extended care paramedics, or as yet unformed roles is urgently needed if paramedicine is to become an effective and valued part of an integrated health system $(31,36-39)$. Based on this Canadian case study, paramedicine programs 
would benefit from an expanded curriculum that includes:

- Generic health and social service education

- Social determinants of health, and other public health topics

- Acute and chronic mental health

- Chronic disease management

- Geriatric emergency medicine

- Wound care

- Understanding of community (sociology)

These 'extra' paramedicine topics are strikingly similar to those identified in the North Central EMS Institute community paramedicine curriculum in the United States that is designed to be offered as a 'postgraduate' course to experienced paramedics (34). In the short-term this is a useful approach to 'up-skilling' existing paramedics taking on community paramedicine roles. However, it does not address the need for a strong underpinning education for all paramedics who may practice as a community paramedic or in other evolving roles, such as extended care or remote area paramedics.

The longer-term approach to broadening paramedicine education is to develop longer, more holistic degree programs that are comparable to the other health professions, such as doctors, nurses, social workers and the allied health professions who work alongside paramedics in a variety of settings $(37,40-42)$. While there exist a considerable number of degree-level paramedicine programs throughout the world $(31,33,43,44)$, the concept is not well established in North America. This reluctance to embrace higher education for paramedics may be related to the historical beginnings of EMS in North America, professional identity and professional status of paramedics, the cost and duration of degree level program relative to the expected remuneration, or the differing registration and accreditation requirements that make the transferability of qualifications problematic $(42,45,46)$.

For community paramedicine there is a broader requirement that the role itself is clearly understood and acknowledged within EMS, amongst the other health professions and by those institutions that both fund and regulate the provision of those health services that constitute the distinctive activities and responsibilities of community paramedics $(23,47)$. Appropriate education of current and aspiring paramedics is a vital step toward a more widespread understanding of the current role of paramedicine, the development of a distinctive body of knowledge, as well as stimulating discussion of the professional identity of the profession now and in the future (48).

\section{CONFLICT OF INTEREST}

The authors declare they have no conflict of interest.

\section{ACKNOWLEDGEMENTS}

We would like to thank the County of Renfrew Paramedic Service and the other health services within the County for their co-operation and assistance with logistics and access to participants. We would also like to thanks La Trobe University for funding the research.

\section{REFERENCES}

1. O'Meara P, Walker J, Stirling C, Pedler D. The rural and regional ambulance paramedic: moving beyond emergency response. Paper presented at: National Rural Health Conference Proceedings2007; Canberra.

2. O'Meara P, Tourle V, Stirling C, Walker J, Pedler D. Extending the Paramedic role in rural Australia: a story of flexibility and innovation. Rural and Remote Health. 2012;12(1978).

3. Council of Ambulance Authorities. When something goes seriously wrong. Melbourne, Australia: Council of Ambulance Authorities;2012.

4. Williams B, Onsman A, Brown T. From stretcher-bearer to paramedic: the Australian paramedics' move toward professionalism. Journal of Emergency Primary Health Care. 2009;7(4):Article No 990346.

5. O'Meara P. The maturation of the paramedic profession through international scholarship. International Journal of Paramedic Practice. 2011a;1(2):2-3.

6. Cooper S, Grant J. New and emerging roles in out of hospital emergency care: A review of the international literature. International Emergency Nursing. 2009;17:90-98.

7. O'Meara P. Community Paramedics: Building a sustainable model of service delivery. NSW Rural Health \& Research Congress. Wagga Wagga2012.

8. Bradley P. Taking Healthcare to the patient 2: A review of 6 years' progress. London: Association of Ambulance Chief Executives;2011.

9. Magin P, Adams J, Sibbritt D, Joy E, Ireland $M$. Experiences of occupational violence in Australian urban general practice: A crosssectional study of GPs. Medical Journal of Australia. 2005;183(7):352-356.

10. Ross J. The Patient Journey Through Emergency Care in Nova Scotia: A Prescription for New Medicine. In: Department of Health, ed: Nova Scotia Government; 2010. 
11. Sheppeard A. Money no carrot for home visits. Medical Observer. 13th March, 2008. ed. On-line.

12. Eburn M, Bendall JC. The provision of Ambulance Services in Australia: a legal argument for the national registration of paramedics. Journal of Emergency Primary Health Care. 2010;8(4):Article 99014.

13. Woollard M. Professionalism in UK paramedic practice. Journal of Emergency Primary Health Care. 2009;7(4):Article No. 990391.

14. Townsend R, Luck M. Protective jurisdiction, patient autonomy and paramedics: the challenges of applying the NSW Mental Health Act. Journal of Emergency Primary Health Care. 2009;7(4):Article No 990375.

15. Emergency Medical Services Chiefs of Canada. Submission to the Standing Committee on Health, December 2011: EMSCC; 2011.

16. Paramedic Association of Canada. National Occupational Competency Profile. 2011; http://paramedic.ca/nocp/.

17. Ruest M, Stitchman A, Day C. Evaluating the impact of 911 calls by an in-home programme with a multidisciplinary team. International Paramedic Practice. 2012;1(4):125-132.

18. Boblin SL, Ireland S, Kirkpatrick H, Robertson K. Using Stake's Qualitative Case Study Approach to Explore Implementation of Evidence-Based Practice. Qualitative Health Research. September 1, 2013 2013;23(9):1267-1275.

19. Morgan PI, Ogbonna E. Subcultural dynamics in transformation: A multi-perspective study of healthcare professionals. Human Relations. 2008;6(1):39-65.

20. Strauss A, Corbin J. Basics of qualitative research: Ground theory procedures and techniques. 2nd ed. Thousand Oaks, CA: Sage; 1998.

21. Natalier K. The Research Question: the heart of the research design. In: Walter $M$, ed. Social Research Methods. Melbourne: Oxford University Press; 2013:40-41.

22. Mays N, Pope C. Qualitative Research: Observational methods in health care settings. British Medical Journal. 1995;311:182.

23. White R, Wingrove G. Principles for Community Paramedicine Programs: National Rural Health Association;2012.

24. Thompson J, Drummond C. Exploring paramedic patient assessment. Journal of Paramedic Practice. 2013;5(6):342-347.

25. Bigham BL, Kennedy SM, Drennan I, Morrison LJ. Expanding Paramedic Scope of Practice in the Community: A Systematic Review of the Literature. Prehospital Emergency Care. 2013;17(3):361-372.
26. Ontario Paramedic Association. Application for Regulation of Paramedics under the Regulated Health Professions Act, 19912013.

27. D'Angelo C. Community Paramedicine: Without the Lights and Sirens. Canadian Paramedicine. 2012;35(2):28-29.

28. Mulholland P, O'Meara P, Walker J, Stirling C, Tourle V. Multidisciplinary Practice in Action: The Rural Paramedic - It's Not Only Lights and Sirens Journal of Emergency Primary Health Care 2009;7(2):Article 990312.

29. Joyce CM, Wainer J, Piterman L, Wyatt A, Archer F. Trends in the paramedic workforce: a profession in transition. Australian Health Review. 2009;33(4):533-540.

30. Lord B. The Development of a Degree Qualification for Paramedics at Charles Sturt University. Journal of Emergency Primary Healthcare. 2003;1(1-2):Article No. 990001.

31. Willis E, Williams B, Brightwell R, O'Meara P, Pointon T. Road-ready paramedics and the supporting sciences curriculum. Focus on Health Professional Education. 2010;11(2):113.

32. Pointon T. Development of professional paramedic education at Flinders University, Adelaide, South Australia. Journal of Emergency Primary Health Care. 2004;2:1-2.

33. Hou X-Y, Rego J, Service M. Review article: Paramedic education opportunities and challenges in Australia. Emergency Medicine Australasia. 2013;25(2):114-119.

34. North Central EMS Institute. Community Paramedic Curriculum 3.0. St Cloud, Minnesota: NCEMSI; 2012:241.

35. Keyes C, Page D, Corry M. How Freedom House has been reborn. Journal of Emergency Medical Services. 2013; March 25, 2013:Article 27365.

36. Walker J. Educating paramedics. In: O'Meara P, Grbich C, eds. Paramedics in Australia: Contemporary challenges of practice. First ed. Sydney: Pearson Education Australia; 2009:152-164.

37. Bowles R, R. From learning activities to the meaning of life: Fostering professionalism in Canadian paramedic education. Journal of Emergency Primary Health Care. 2009;7(4):Article No 990392.

38. Council of Ambulance Authorities. Guidelines for the Assessment and Accreditation of Entry-level Paramedic Education Programs. Adelaide: Council of Ambulance Authorities Inc.;2010.

39. Michau R, Roberts G, Williams B, Boyle M. An investigation of theory-practice gap in undergraduate paramedic education. BMC Medical Education. 2009;9(1):Article No. 23.

40. Ambulance Service Association. The future role and education of paramedic ambulance service personnel (emerging concepts)2000. 
41. Cooper S. Contemporary UK paramedical training and education. How do we Train? How should we educate? Emerg Med J. 2005;22:375-379.

42. Leggio WJ. Is the Bar for Entry into the Practice of EMS Too Low? A Reflection from the Inside and Outside. DOMAIN3. 2013;Spring/Summer:18-24.

43. Hallikainen J, Väisänen $O$, Rosenberg $P H$, Silfvast T, Niemi-Murola L. Interprofessional education of medical students and paramedics in emergency medicine. Acta Anaesthesiologica Scandinavica. 2007;51:372-377.

44. Margolis G. The Role of Bachelor's Degree Emergency Medical Services Programs in the Professionalization of Paramedicine: University of Pittsburgh; 2005.

45. King JA. NHTSA Notes Commentary: EMS Education Agenda for the Future: Flexner Revisited. Annals of Emergency Medicine. 2000;35(6):624-625.

46. National Association of EMS Educators. Position Statement: EMS education agenda for the future: a systems approach. Prehospital Emergency Care. October/December 2000;4(4):365-366.

47. Patterson DG, Skillman SM. A National Agenda for Community Paramedicine Research. Seattle, WA: WWAMI Rural Health Research Centre, University of Washington;2012.

48. O'Meara P. So how can we frame our identity? Journal of Paramedic Practice. 2011b;3(2):5. 